\title{
Noise-robust method for image segmentation
}

\author{
Ivana Despotović, Vedran Jelača, Ewout Vansteenkiste and Wilfried Philips \\ Ghent Univesity, Department of Telecommunications and Information Processing, \\ TELIN-IPI-IBBT, \\ Sint-Pietersnieuwstraat 41, 9000 Ghent, Belgium \\ \{ivek, vjelaca, ervsteen, philips\}@telin.ugent.be \\ http://telin.ugent.be/ipi/drupal
}

\begin{abstract}
Segmentation of noisy images is one of the most challenging problems in image analysis and any improvement of segmentation methods can highly influence the performance of many image processing applications. In automated image segmentation, the fuzzy c-means (FCM) clustering has been widely used because of its ability to model uncertainty within the data, applicability to multi-modal data and fairly robust behaviour. However, the standard FCM algorithm does not consider any information about the spatial image context and is highly sensitive to noise and other imaging artefacts. Considering above mentioned problems, we developed a new FCM-based approach for the noise-robust fuzzy clustering and we present it in this paper. In this new iterative algorithm we incorporated both spatial and feature space information into the similarity measure and the membership function. We considered that spatial information depends on the relative location and features of the neighbouring pixels. The performance of the proposed algorithm is tested on synthetic image with different noise levels and real images. Experimental quantitative and qualitative segmentation results show that our method efficiently preserves the homogeneity of the regions and is more robust to noise than other FCM-based methods.
\end{abstract}

Key words: Image segmentation, Noise, Fuzzy clustering, Fuzzy CMeans, Spatial information

\section{Introduction}

Image segmentation is often a critical component in many image applications and is typically used to partition images into a set of non-overlapping, homogeneous regions with similar attributes such as intensity, texture, depth, color, etc. The diversity of image applications have led to the development of various segmentation techniques that vary in both algorithmic approach and the quality and nature of the segmentation produced. Some applications require the image to be segmented in details, while others require coarse homogeneous regions. Since unsupervised fuzzy clustering is one of the most commonly used methods for automatic image segmentation [1,2] and has been successfully applied in fields such as astronomy, geology, medical and molecular imaging, it will be considered in this paper. 
Fuzzy clustering methods involve the idea of partial membership and allow pixels to belong to multiple classes with certain degree. This idea is very important in applications where uncertainty, poor contrast, limited spatial resolution and noise are present (e.g. satellite and medical images). Among fuzzy clustering methods, the fuzzy c-means (FCM) algorithm [3] is the most popular one. However, the conventional FCM algorithm has a drawback, it classifies pixels in the feature space without considering their spatial distribution in the image and thus it is highly sensitive to noise.

To overcome above mentioned problem and reduce segmentation errors, many extensions of the FCM algorithm have been proposed [4-9]. The most common approach is to include spatial neighbourhood information by modifying the FCM objective function $[4,5]$ or a similarity measure between cluster centres and elements [6]. Ahmed et al. [4] modified the objective function of the standard FCM algorithm to allow the immediate neighbours of the pixel to influence its labelling. On the other hand, to keep the continuity from the FCM algorithm, Shen et al. [6] introduced a new similarity measure that depends on spatial neighbourhood information, where the degree of the neighbourhood attraction is optimized by a neural network. Beside these modifications, there are also other methods that can be used to enhance the FCM performance. For example, one can combine the pixel-wise classification with preprocessing (noise cleaning in the original image) $[7,10]$ and post-processing (noise cleaning on the classified data). Xue et al. [10] proposed an algorithm where they firstly denoise images, then classify the pixels using the standard FCM method and finally refine the segmentation with post-processing filtering. All of these methods can reduce the noise to a certain extent, but still have some drawbacks such as increased complexity $[4,6,9]$ and image smoothing $[7,10]$ that can result in loss of important image details.

In this paper, we present a new noise-robust FCM-based algorithm for image segmentation. Our algorithm iteratively integrates spatial neighbourhood information of the image elements (pixels) into both the similarity measure and the membership function. The spatial information depends on the relative location, intensities and membership degree values of the neighbouring pixels. The efficiency of our method is tested on synthetic and real images with different noise levels. Experimental results indicate that our method successfully reduces the effect of noise and biases the algorithm toward homogeneous clustering.

The paper is organized as follows. In Section 2, we explain the standard FCM method and our modified FCM algorithm. Experimental results together with comparison with other methods are presented and discussed in Section 3. Finally, we conclude this paper in Section 4.

\section{Method}

\subsection{FCM algorithm}

The FCM algorithm, initially developed by Dunn and later generalized by Bezdek [3], is an iterative, unsupervised, soft classification method that can obtain much 
more information from the original image than hard segmentation methods (e.g. k-means). While hard segmentation methods classify pixels to belong exclusively to one class, FCM allows pixels to belong to multiple classes with different membership degrees.

Let $X=\left\{\mathbf{x}_{j}, j=1,2, \ldots, N \mid \mathbf{x}_{j} \in \mathbb{R}^{q}\right\}$ represent feature vectors of the image with $N$ pixels that needs to be partitioned into $C$ classes, where every component of the vector $\mathbf{x}_{j}$ represents a feature of the image at position $j$ and $q$ is the dimension of the feature vector. The FCM clustering algorithm is based on minimizing the following objective function:

$$
J_{m}=\sum_{i=1}^{C} \sum_{j=1}^{N} u_{i j}^{m} D_{i j},
$$

where $u_{i j}$ is the membership function of the feature $\mathbf{x}_{j}$ belonging to the $i$-th cluster, $m$ is the weighting exponent that controls the fuzziness of the resulting partition (most often is set to $m=2$ ) and $D_{i j}=d^{2}\left(\mathbf{x}_{j}, \mathbf{v}_{i}\right)$ is the similarity measure between $\mathbf{x}_{j}$ and the $i$-th cluster center $\mathbf{v}_{i}$. The most commonly used similarity measure is the squared Euclidean distance:

$$
D_{i j}=d^{2}\left(\mathbf{x}_{j}, \mathbf{v}_{i}\right)=\left\|\mathbf{x}_{j}-\mathbf{v}_{i}\right\|^{2} .
$$

The objective function $J_{m}$ (Eq. (1)) is minimized under the following constraints:

$$
u_{i j} \in[0,1], \quad \sum_{i=1}^{C} u_{i j}=1, \forall j \text { and } 0<\sum_{j=1}^{N} u_{i j}<N, \forall i
$$

where low membership values are assigned to pixels far from the cluster centroid, and high membership values to pixels close to the cluster centroid. Considering the constraints $u_{i j}$ from Eq. (3) and calculating the first derivatives of $J_{m}$ with respect to $u_{i j}$ and $\mathbf{v}_{i}$ and setting them to zero, results in two following conditions for minimizing $J_{m}$ :

$$
u_{i j}=\left[\sum_{k=1}^{C}\left(\frac{D_{i j}}{D_{k j}}\right)^{\frac{1}{m-1}}\right]^{-1}
$$

and

$$
\mathbf{v}_{i}=\frac{\sum_{j=1}^{N} u_{i j}^{m} \mathbf{x}_{j}}{\sum_{j=1}^{N} u_{i j}^{m}},(i=1,2, \ldots, C) .
$$

The FCM algorithm iteratively optimizes $J_{\mathrm{m}}$, by evaluating Eq. (4) and Eq. (5), until the following stop criterion is satisfied:

$$
\max _{i \in[1, C]}\left\|\mathbf{v}_{i}^{(l)}-\mathbf{v}_{i}^{(l+1)}\right\|_{\infty}<\epsilon,
$$

where $l$ is the iteration index and $\|\cdot\|_{\infty}$ is the $L_{\infty}$ norm. Once a membership value $u_{i j}$ for each class $i$ is assigned to each pixel $j$, a defuzzification of the fuzzy 
clusters $\left\{F_{k}\right\}_{k=1}^{C}$ into its crisp version $\left\{H_{k}\right\}_{k=1}^{C}$ is done by assigning the pixel to the class with the highest membership value as follows:

$$
\max _{i \in[1, C]}\left(u_{i j}\right)=u_{k j} \Longrightarrow \mathbf{x}_{j} \in H_{k}
$$

The main drawback of the standard FCM for image segmentation is that the objective function does not take into account any spatial information and deals with the pixels as separate points. Therefore, the standard FCM algorithm is sensitive to outliers and very often those pixels are wrongly classified. To illustrate this, we consider an example shown in Fig. 1, where we have a simple synthetic image with two classes (grey background and white foreground) Fig. 1a. The white foreground and the black background are corrupted by the noise pixels, which have the same intensity value as the opposite class, the background and the foreground respectively. The segmentation result using the FCM Fig. 1b is affected by the noise pixels, while the desired segmentation result is shown in Fig. 1c.

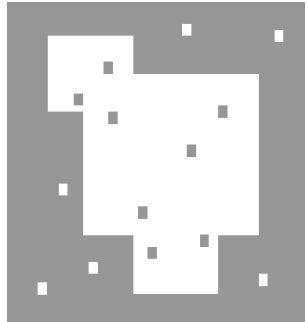

(a)



(b)

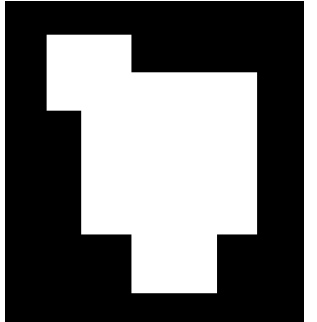

(c)

Fig. 1. Example: (a) a synthetic image with a noise, (b) the FCM segmentation result and (c) the expected segmentation result.

\section{$2.2 \quad$ Proposed method}

One of the important characteristics of the image is that majority of its neighbouring pixels have similar feature values and the probability that they belong to the same cluster is great. Therefore, to improve the performance and overcome the limitation of the standard FCM algorithm, we considered the spatial neighbourhood information in our method.

If we look at the objective function of the FCM algorithm (Eq. (1)) and its two necessary conditions for the convergence (Eq. (4) and Eq. (5)), we can conclude that the segmentation result is significantly influenced by membership values $u_{i j}$ and the choice of the similarity measure $D_{i j}$. The novelty of our method is that in each iteration of the algorithm we modify both the similarity measure and the membership values, in two separate steps, using the spatial 
information of the neighbouring pixels. The new similarity measure includes the intensity and distance of the neighbouring pixels, while the new membership function is calculated using the membership degree values of the neighbouring pixels weighted by their spatial position in the image. In the following lines we explain these two steps in more details.

Firstly, we define the spatially dependent similarity measure as follows:

$$
D_{i j}=\left\|\mathbf{x}_{j}-\mathbf{v}_{i}\right\|^{2}\left(1-\alpha S_{i j}\right),
$$

where $S_{i j}$ represents the spatial neighbourhood information and $\alpha \in[0,1]$ is the parameter that controls the relative importance of the neighbourhood attraction. If $\alpha=0, D_{i j}$ is the squared Euclidean distance and we have the standard FCM. The spatial information $S_{i j}$ depends on the feature attraction $a_{j r}$ (pixel intensities) and the distance attraction $d_{j r}$ (relative location of neighbouring pixels), and is defined as:

$$
S_{i j}=\frac{\sum_{r=1}^{N_{r}} u_{i r} a_{j r} d_{j r}^{-1}}{\sum_{r=1}^{N_{r}} a_{j r} d_{j r}^{-1}},
$$

where $N_{r}$ is the number of neighbours surrounding the element $\mathbf{x}_{j}$ in a square window $\Omega_{j}$, and $u_{i r}$ is the membership degree of the neighbouring element $\mathbf{x}_{r}$ to the cluster $i$. If we define the neighbourhood configuration $\Omega_{j}$ as an $n \times n$ square window with the central element $\mathbf{x}_{j}$, then $r=n^{2}-1$ and $\Omega_{j}=\left\{\mathbf{x}_{r} \mid r=\right.$ $\left.1,2, \ldots, n^{2}-1\right\}$. Feature attraction $a_{j r}$ is defined as the absolute intensity difference between $\mathbf{x}_{j}$ and its neighbour $\mathbf{x}_{r}$

$$
a_{j r}=\left|\mathbf{x}_{j}-\mathbf{x}_{r}\right| \text {. }
$$

The distance attraction $d_{j r}$ is the squared Euclidean distance between the coordinates of elements $\mathbf{x}\left(p_{j}, q_{j}\right)$ and $\mathbf{x}\left(p_{r}, q_{r}\right)$

$$
d_{j r}=\left(p_{j}-p_{r}\right)^{2}+\left(q_{j}-q_{r}\right)^{2} .
$$

Secondly, after modifying the similarity measure, we calculate the membership values using Eq.(4). Then, we use the spatial neighbourhood information again to calculate the new spatially dependent membership values in the following way:

$$
u_{i j}^{*}=\frac{u_{i j} M_{i j}^{2}}{\sum_{k=1}^{C} u_{k j} M_{k j}^{2}}
$$

and

$$
M_{i j}=\sum_{r=1}^{N_{r}} u_{i r} d_{j r}^{-1},
$$

where $u_{i j}^{*}$ is the new spatially dependent membership value, $C$ is the number of classes, $N_{r}$ is the number of neighbours surrounding the element $\mathbf{x}_{j}$ and $M_{i j}$ is the spatial membership function that represents the probability that element $\mathbf{x}_{j}$ belongs to the cluster $i$. 
In both equations, Eq. (9) and Eq. (13), the reciprocal of the distance $d_{j r}^{-1}$ is used because the neighbours $\mathbf{x}_{r}$ close to the central element $\mathbf{x}_{j}$ should more influence the result, while further neighbours should be less important.

The idea behind this new integration of spatial information in the FCM algorithm is as follows. Consider the local $n \times n$ neighbourhood where the central element $\mathbf{x}_{j}$ has large intensity differences with the closest neighbouring elements $\mathbf{x}_{r}$, which have similar intensities as the cluster center $\mathbf{v}_{i}$. After running the standard FCM algorithm, the neighbouring elements will be classified in a cluster $i$, while the central element will be in a different cluster. However, if we consider spatial information and calculate the neighbourhood attraction $S_{i j}$, which will be large in this case and the expression $\left(1-\alpha S_{i j}\right)$ will be small for $\alpha \neq 0$, the new spatially dependent similarity measure will be smaller than before. That means that after one iteration of the algorithm the central element $\mathbf{x}_{j}$ will be attracted to the neighbouring cluster $i$. Next, if we calculate the new spatial membership function $M_{i j}$ and update the membership values, we will get that in a homogeneous regions the new membership values stay unchanged, while for a noisy pixel the new membership value is influenced by the labels of its neighbouring pixels. In our case, the central element $\mathbf{x}_{j}$ is then even stronger attracted to the cluster $i$. If the neighbourhood attraction $S_{i j}$ and $M_{i j}$ are continuously large till the end of the algorithm, the central element $\mathbf{x}_{j}$ will be forced to belong to the cluster $i$ despite being dissimilar to it. Precisely, this property biases the algorithm towards homogeneous clustering.

The outline of the proposed algorithm is:

Step1. Set the number of clusters $C$, degree of fuzziness $m$, stop criterion $\epsilon$ and neighbourhood size.

Step2. Initialize the centres of the clusters $\mathbf{v}_{i} \mid i=1,2, \ldots, C$ and using FCM calculate $u_{i j}$.

Step3. Calculate the spatially dependent similarity measure Eq. (8).

Step4. Update $u_{i j}$ using the new similarity measure Eq. (4).

Step5. Calculate the new membership values $u_{i j}^{*}$ using the spatial membership function Eq. (12).

Step6. Update $\mathbf{v}_{i}$ using new membership values $u_{i j}^{*}$ Eq. (5).

Repeat steps 3-6 until the stop criterion Eq.(6) is satisfied.

As with all clustering algorithms, the segmentation result may highly depend on the choice of parameter values used for initialization. Therefore, we use intensity-based thresholding [11] to reliably initialize the cluster centres.

\section{Results}

In this section, the experimental results of our algorithm to synthetic and real images are presented. For all experiments we set the weighting exponent $m=2$, the stop criterion $\epsilon=0.01$, the neighbourhood size $3 \times 3$ and the parameter that controls the effect of the neighbours $\alpha=1$.

To investigate the sensitivity of the proposed method to noise and to show the quantitative comparative results with other FCM-based methods $[3,4,6,10]$, we 
use the synthetic image $($ size $128 \times 128)$ shown in Fig. $2 \mathrm{~g}$. It contains four-class pattern with three different shapes and is corrupted by zero mean Gaussian noise (Fig. 2a), where Signal-to-Noise Ratio (SNR) between the original and noisy image is $12 \mathrm{~dB}$.

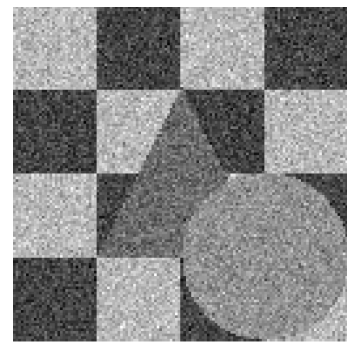

(a)

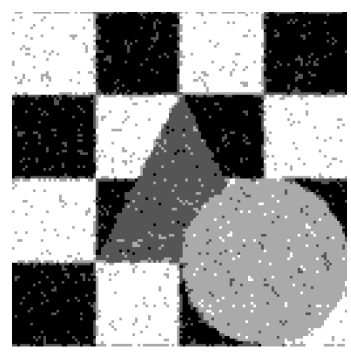

(d)



(b)

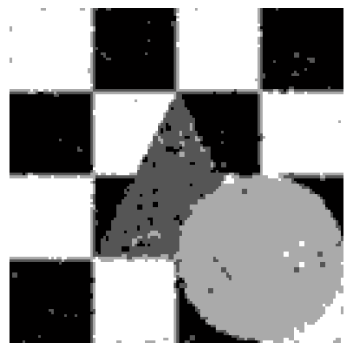

(e)

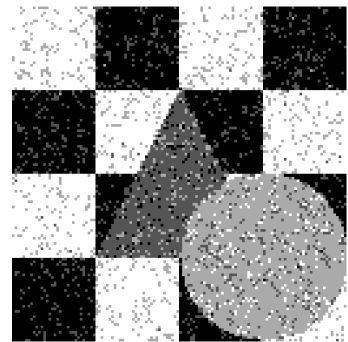

(c)

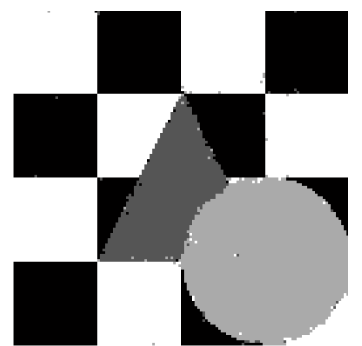

(f)



(g)

Fig. 2. Results comparison of the five segmentation methods on a synthetic image with four grey levels and three different shapes: (a) the image corrupted by zero mean Gaussian noise (SNR=12dB); (b) the FCM method [3]; (c) Shen et al. method [6]; (d) Ahmed et al. method [4]; (e) Xue et al. method [10]; (f) the segmentation result of our algorithm; (g) the "ground truth" - original synthetic image.

As can be seen in Fig. 2b, the FCM algorithm [3] can not correctly classify four classes and is highly sensitive to outliers. The methods of Shen et al. [6] and Ahmed et al. [4], with results given in Fig. 2c and Fig. 2d respectively, although 
incorporating spatial information, are not sufficient enough to segment the image with very low SNR. The method from Xue et al. [10], which uses image filtering before and after the segmentation, also does not give satisfactory result and still contains artefacts and additional edge blurring (Fig. 2e). However, the result of our method Fig. 2f shows good performance and achieves the best segmentation result comparing with the "ground truth" image Fig. $2 \mathrm{~g}$.

In order to obtain a quantitative comparison, we plot the validation results of five methods for different noise levels in Fig. 3. The similarity index $\rho$, used for the comparison and quantitative evaluation, is the Dice coefficient:

$$
\rho=\frac{2\left|A_{i} \bigcap B_{i}\right|}{\left|A_{i}\right|+\left|B_{i}\right|}
$$

where $A_{i}$ and $B_{i}$ denote the set of pixels labelled into $i$ by the "ground truth" and our method respectively, and $\left|A_{i}\right|$ denotes the number of elements in $A_{i}$. In our experiment, the results for $\rho$ are averaged over all four classes.

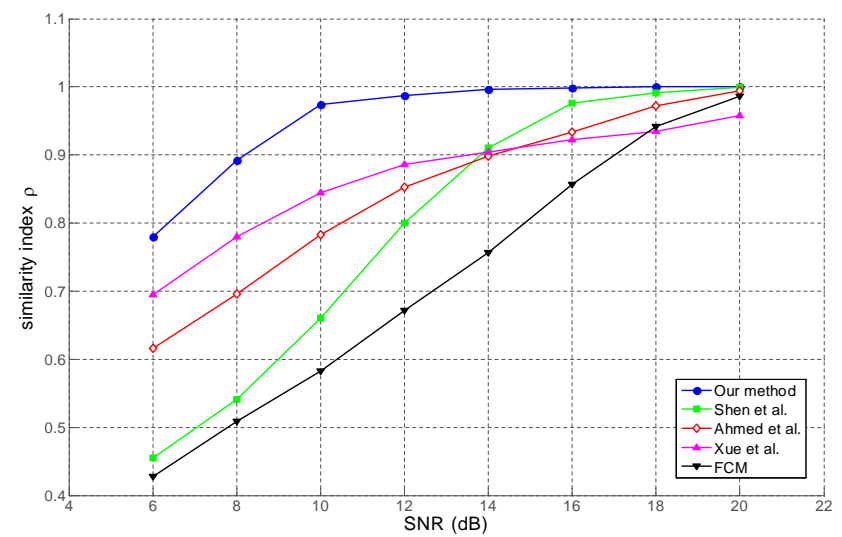

Fig. 3. Validation result for different noise levels. Comparison of FCM [3], Ahmed et al. [4], Xue et al. [10], Shen et al. [6] and our algorithm. From the graph, we can clearly see that our algorithm outperforms the standard FCM and popular spatial clustering variants, especially for the lower SNR.

From the Fig. 3 we can clearly see that our algorithm outperforms other FCM-based methods and acquires the best segmentation performance for all noise levels.

The performance of our algorithm is also demonstrated on four real images corrupted with noise: the cameraman, a house, a CT (Computer Tomography) image of the liver and an MRI (Magnetic Resonance Imege) of the brain. Segmentation results of the FCM and our algorithm are shown in the figure Fig. 4. 

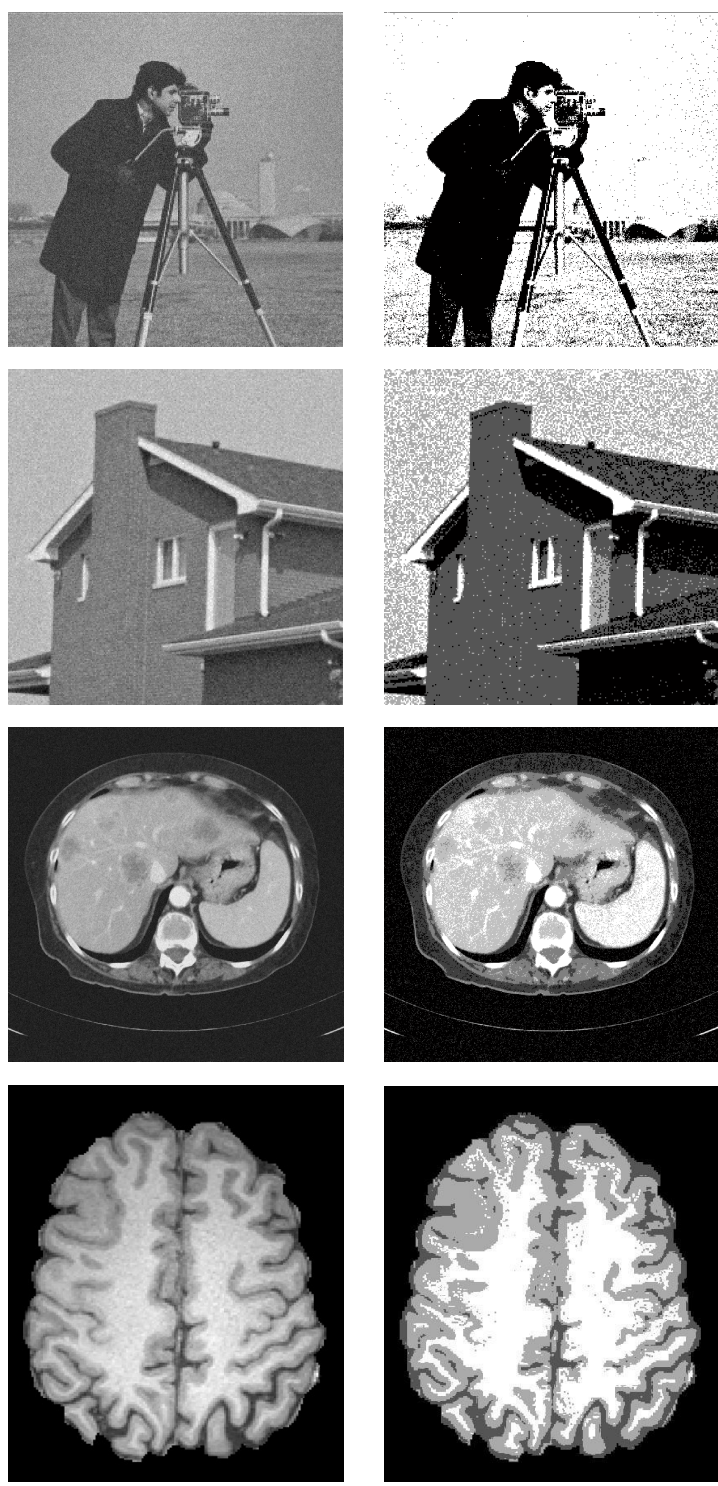

(a)
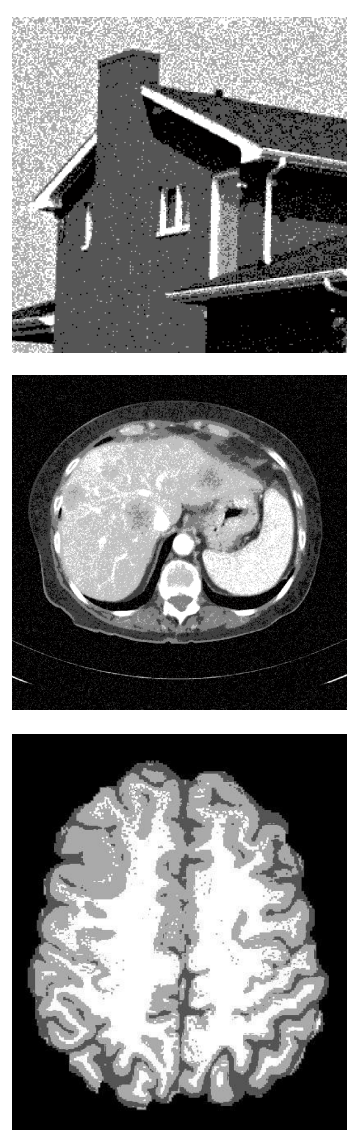

(b)


(c)

Fig. 4. Segmentation results on four real images corrupted with noise. The first column (a) shows noisy images, the second column (b) shows the results using FCM algorithm and the third column (c) shows the segmentation performance of our algorithm. The first two images, the cameraman and a house, are segmented in two and four labels respectively. Next, the CT image of the liver is segmented in five labels. Finally, the MRI of the brain, with a lesion on the upper-left side, is segmented in three labels. Comparing the second and third columns, it is clear that our method is effective and gives noise-free segmentation. 


\section{Conclusion}

We have presented a new algorithm for unsupervised and automatic segmentation of images corrupted with noise. Our method is based on the FCM clustering approach, feature space and spatial contextual information of the neighbouring pixels in the image. The quantitative and qualitative experimental results for simulated and real images show that our method is very efficient and can effectively reduce the sensitivity of fuzzy segmentation algorithms to noise, without blurring the image. Also, it is good at resolving classification ambiguity for data in the overlapping region of two clusters. Our method could be useful in applications such as image texture segmentation, medical image segmentation and multispectral image segmentation, where spatial contextual information is important.

\section{References}

1. J. V. de Oliveira and W. Pedrycz, Advances in Fuzzy Clustering and its Applications, John Wiley \& Sons, Inc., New York, NY, USA, 2007.

2. N. Pal and S. Pal, "A review on image segmentation techniques," Pattern Recognition, vol. 26, pp. 1277-1294, 1993.

3. J. C. Bezdek, Pattern Recognition with Fuzzy Objective Function Algorithms, Plenum Press, New York, 1981.

4. M. N. Ahmed, S. M. Yamany, N. Mohamed, A. A. Farag, and T. Moriaty, "A modified fuzzy c-means algorithm for bias field estimation and segmentation of MRI data," IEEE Trans. Med. Imag., vol. 21, pp. 193-199, 2002.

5. D. Zhang and C. Chen, "A novel kernelized fuzzy c-means algorithm with application in medical image segmentation," Artificial Inteligence in Medicine, vol. 32, pp. 37-50, 2004.

6. S. Shen, W. Sandham, M. Granat, and A. Sterr, "MRI fuzzy segmentation of brain tissue using neighborhood attraction with neural-network optimization," IEEE Trans. Inf. Technology in Biomedicine, vol. 9, pp. 459-467, 2005.

7. W. Cai, S. Chen, and D. Zang, "Fast and robust fuzzy c-means clustering algorithms incorporating local information for image segmentation," Pattern Recognition, vol. 40, pp. 825-838, 2007.

8. A. Z. Cao and Q. Song, "Robust information clustering for automatic breast mass detection in digitized mammograms," Comput. Vision Image Understanding, vol. 109, pp. 87-96, 2008.

9. Z. M. Wang, Y. C. Soh, Q. Song, and K. Sim, "Adaptive spatial informationtheoretic clustering for image segmentation," Pattern Recognition Letters, vol. 42, pp. 2029-2044, 2009.

10. J.-H. Xue, A. Pižurica, W. Philips, E. Kerre, R. Van de Walle, and I. Lemahieu, "An integrated method of adaptive enhancement for unsupervised segmentation of MRI brain images," Pattern Recognition Letters, vol. 24, pp. 2549-2560, 2003.

11. S. S. Reddi, S. F. Rudin, and H. R. Keshavan, "An optical multiple threshold scheme for image segmentation," IEEE Trans. System Man Cybernet., vol. 14, pp. 661-665, 1984.

12. R. Unnikrishnan, C. E. Pantofaru, and M. Hebert, "Toward objective evaluation of image segmentation algorithms," IEEE Trans. Pattern Anal. Machine Intell., vol. 29, pp. 929-943, 2007. 